\title{
Non-Random Interaction of Vacancies with Atoms during Interdiffusion and Ionic Conductivity in Materials
}

\author{
I.V. Belova ${ }^{1, a}$ and G.E. Murch ${ }^{1, b}$ \\ ${ }^{1}$ Diffusion in Solids Group, \\ Centre for Geotechnical and Materials Modelling, \\ School of Engineering \\ The University of Newcastle, Callaghan, NSW 2308, Australia
}

arina.Belova@newcastle.edu.au, ${ }^{\text {b } G r a e m e . M u r c h @ n e w c a s t l e . e d u . a u ~}$

Keywords: Interdiffusion, intrinsic diffusion, ionic conductivity, vacancy-wind effect, correlation factors.

\begin{abstract}
The non-random interaction of vacancies with atoms during interdiffusion and ionic conductivity is referred to as the vacancy-wind effect. This effect, first discovered by the late Dr John Manning, is a subtle phenomenon arising from the non-random distribution of vacancies with respect to a given moving atom within a net flux of vacancies. Recently, a good deal of progress has been made in determining accurate expressions for vacancy-wind factors in binary and ternary alloys, and in mixed cation ionic systems. The present paper provides an overview of these recent findings and puts them into a broader and historical context.
\end{abstract}

\section{Introduction}

Some forty years ago, the late Dr John Manning discovered the phenomenon of the vacancywind (sometimes called the vacancy-flow) effect in solid state diffusion; see, for example [1,2] and references therein. Manning showed how this phenomenon arises naturally in any diffusion situation when there is a net flux of vacancies and two or more species of atom on the same lattice jump with the vacancies at different rates. (A net flux of vacancies normally occurs in interdiffusion in binary and ternary etc alloys and also in the d.c. ionic conductivity in ionic conductors.) In some cases, even the direction of an atomic flux can be reversed because of the vacancy-wind. When it was introduced, the vacancy-wind effect was a little controversial and it was not until computer simulation [3] verified the phenomenon that the vacancy-wind effect became universally accepted. The vacancy-wind effect, like correlations that occur generally in solid state diffusion, is intimately connected with the existence of non-zero off-diagonal phenomenological transport coefficients or $L$ coefficients. In general, if the off-diagonal phenomenological coefficients are zero (this is equivalent to the Darken approximation in interdiffusion [4]), there is no vacancy-wind effect. The only known apparent exception to this is the case of diffusion by way of six-jump-cycles in intermetallic compounds [5]. It is worth noting that the terminology 'vacancy-wind' is actually somewhat of a misnomer since the general effect on the atomic fluxes can also occur as a result of atomic diffusion taking place via other defects (such as dumb-bell interstitials) in a net flux of such defects.

The vacancy-wind effect is conveniently manifested as deviations in the 'expected' flow of tracer atoms. Then the vacancy-wind effect is expressed in terms of 'vacancy-wind factors'. These factors, of which there are quite a number in the literature, appear in expressions relating collective transport quantities, such as interdiffusion coefficients and ionic conductivities, and the corresponding tracer diffusion coefficients of the various mobile components. Much of the initial understanding of the behaviour of vacancy-wind factors was provided by Manning himself using two well-known models: the five-frequency model and the random alloy model [1] and his own diffusion kinetics formalisms that were developed to describe diffusion in these models [1]. 
Recently, a good deal of progress has been made in determining rather accurate expressions for the various vacancy-wind factors with the random mixing (alloy) model and its application to interdiffusion in binary and ternary alloys and mixed cation ionic systems. The present paper provides an overview of these recent findings and puts them into a broader and historical context.

\section{Vacancy-Wind Factors in Interdiffusion in Binary Alloys}

The vacancy-wind effect is almost certainly best known for the case of interdiffusion in binary alloys. In this case, it is embodied in the vacancy-wind factor $S$ appearing in what is usually called the Darken-Manning Equation. This important equation relates the interdiffusion coefficient, $\widetilde{D}$, the two tracer diffusion coefficients $D^{*}{ }_{A}$ and $D^{*}{ }_{B}$ for the atomic components $A$ and $B$ respectively and the thermodynamic factor $\phi[1]$ :

$$
\widetilde{D}=S\left(c_{B} D_{A}^{*}+c_{A} D_{B}^{*}\right) \phi
$$

where $c_{A}$ and $c_{B}$ are the atomic compositions (site fractions) of $A$ and $B$ respectively. In the sense that it can be derived using the Onsager flux equations of irreversible thermodynamics, Eq. 1 as written here is exact for any interdiffusion process that makes use of vacancies in a binary alloy. There is a (hidden) assumption that the sources and sinks of vacancies are sufficiently numerous so that vacancy under-saturation and super-saturation do not occur in the diffusion zone. $S$ can be expressed in terms of the correlated parts (called collective correlation factors) of the phenomenological coefficients (the $L_{i j}$ ) and the correlated parts (called tracer correlation factors) of the tracer diffusion coefficients:

$$
S=\frac{c_{B} f_{A A}+c_{A} g f_{B B}-c_{B} g f_{A B}^{(B)}-c_{A} f_{A B}^{(A)}}{c_{B} f_{A}+c_{A} g f_{B}}
$$

where $f_{A A}, f_{B B}, f_{A B}^{(A)}, f_{A B}^{(B)}$ are called the collective correlation factors and $f_{A}$ and $f_{B}$ are the more familiar tracer correlation factors, $g=n_{B} / n_{A}$ where $n_{A}$ and $n_{B}$ are the numbers of jumps of an $A$ or a $B$ atom in time $t$. It should be noted that this expression for $S$ does not appear in the early literature of the vacancy-wind effect because collective correlation factors were not introduced into the literature until about 1984 [6].

If we choose a particular model for the binary alloy, then expressions can then be developed for $S$ in terms of measurable quantities, principally the tracer diffusion coefficients. For the random alloy model (this is a model in which it is assumed that the atoms and vacancies are randomly mixed) Manning was able to express the $S$ factor in terms of the two tracer diffusion coefficients $D^{*}{ }_{\mathrm{A}}, D^{*}{ }_{\mathrm{B}}$ and $f_{0}$, the geometric tracer correlation factor for the lattice (e.g. $f_{0}=0.78145$ for the f.c.c. lattice). Manning's expression is [1]:

$$
S=1+\frac{2 c_{A} c_{B}\left(D_{A}^{*}-D_{B}^{*}\right)^{2}}{M_{0}\left(c_{B} D_{A}^{*}+c_{A} D_{B}^{*}\right)\left(c_{A} D_{A}^{*}+c_{B} D_{B}^{*}\right)}
$$


where $M_{0}=2 f_{0} /\left(1-f_{0}\right)$. Although developed for the random alloy, it has been shown by Monte Carlo simulation that Eq. 1, with $S$ given by Eq. 3, is surprisingly powerful and provides quite a reasonable approximation even in intermetallic compounds of quite complex structures provided that there is always a good deal of antistructural disorder; see, for example, [7]. Eq. 3 can also be rewritten in terms of the tracer correlation factors of the atomic components and the vacancy-atom exchange frequencies for $\mathrm{A}$ atoms and $\mathrm{B}$ atoms $w_{A}$ and $w_{B}$ :

$$
S=\frac{\left(f_{A} / f_{0}\right) f_{B} c_{A}\left(w_{A}-w_{B}\right)-w_{A}}{f_{B} c_{A}\left(w_{A}-w_{B}\right)-w_{A}}=\frac{\left(f_{B} / f_{0}\right) f_{A} c_{B}\left(w_{B}-w_{A}\right)-w_{B}}{f_{A} c_{B}\left(w_{B}-w_{A}\right)-w_{B}}
$$

Eq. 3 has become so closely identified with the Darken-Manning Equation (Eq. 1) that in some instances in the literature it has been implied that $S$ is always given simply by Eq. 3. This is not the case. We stress that Eq. 3 is purely an approximation for $S$ using Manning's approximate diffusion kinetics analysis of the random alloy model. Eq. 3 gives for the limits of $S$ : $1.0 \leq S \leq f_{0}^{-1}$. Accordingly, for the f.c.c. lattice (where $f_{0}$ is given by 0.78145 ), $S$ is seen to take the upper limit of 1.27967.

The self-consistent theory of Moleko, Allnatt and Allnatt (MAA) [8], which has been developed since Manning's pioneering contributions, also describes diffusion kinetics in the random alloy model. The MAA formalism has been shown by recent extensive Monte Carlo simulation to provide an almost exact treatment for tracer correlation factors and collective correlation factors for the random alloy model and therefore all aspects of tracer and collective diffusion in the random alloy. In the case of the vacancy-wind factor $S$, an expression for $S$ from the MAA formalism is unfortunately not available in closed form and must be calculated numerically [9]. The MAA results for $S$ are in good agreement with those of Manning for values of the atom-vacancy exchange frequency ratio within about a factor of five of unity. MAA gives unity as the lower limit for $S$ (the same as the Manning approximation). However, instead of the Manning upper limit of $f_{0}^{-1}$ as seen above, at compositions near the percolation threshold composition $c_{\mathrm{A}}=f_{0}, S$ approaches infinity when the ratio of the vacancy atom exchange frequencies $w_{A} / w_{B}$ approaches zero, see Fig. 1 . This finding has also been verified, where possible, by Monte Carlo simulation [9]. However, it should be noted that for practical purposes, even when the ratio of the vacancy-atom exchange frequencies of the two atomic components is as small as $1 / 100$ (and this is relatively rare in practice), $S$ only increases to approximately two at the percolation composition. 


\section{Vacancy-Wind Factors in Intrinsic Diffusion in Binary Alloys}

There are also two closely related expressions to the Darken-Manning Equation (Eq. 1), that relate the two intrinsic diffusion coefficients $D_{A}$ and $D_{B}$ (these diffusion coefficients are obtained from the interdiffusion coefficient and the marker or Kirkendall shift) to the two tracer diffusion coefficients and the thermodynamic factor [1]:

$$
D_{A}=\phi s_{A} D_{A}^{*} ; \quad D_{B}=\phi s_{B} D_{B}^{*}
$$

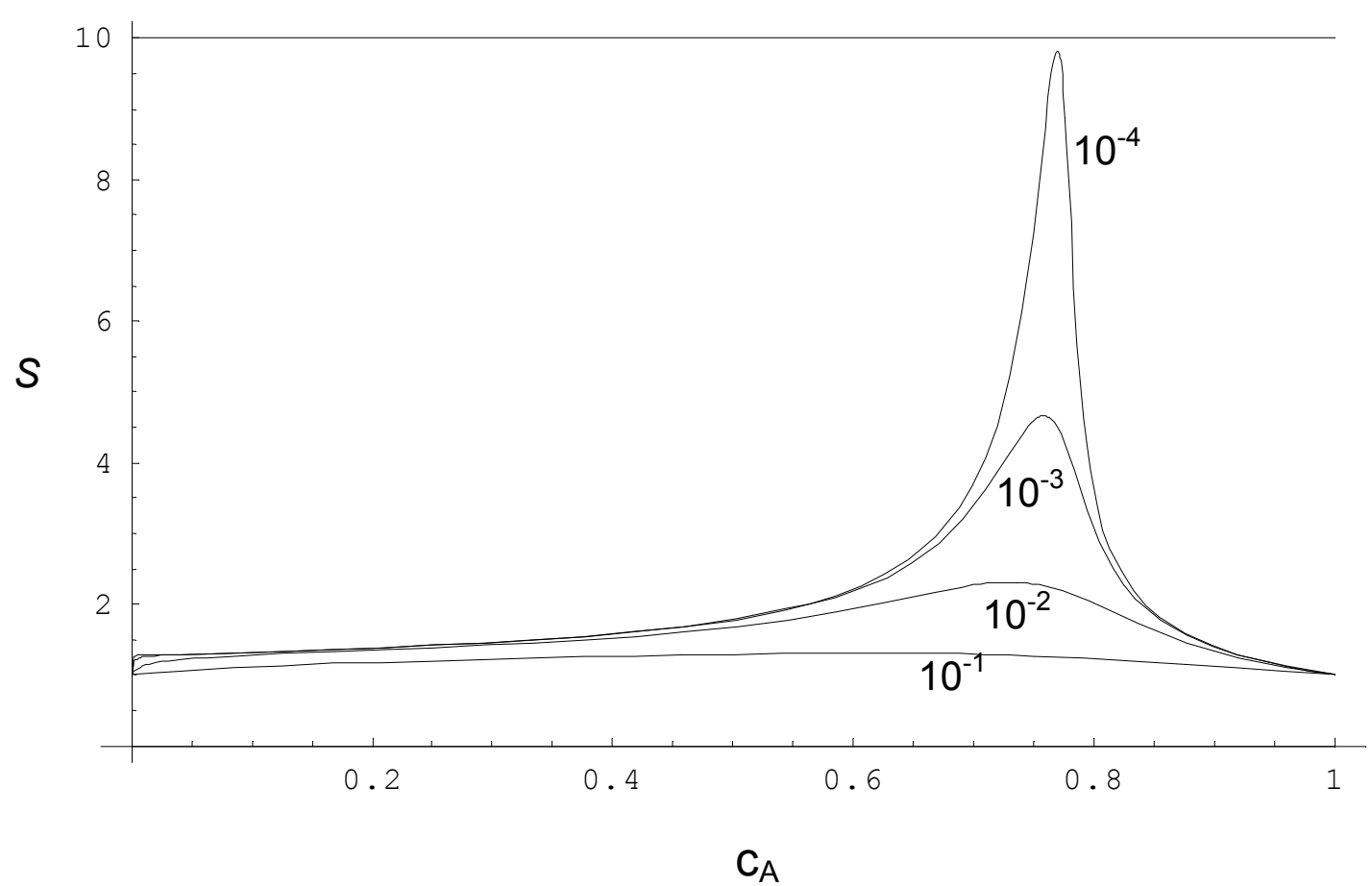

Figure 1. The vacancy-wind factor $S$ (calculated using the MAA diffusion kinetics theory for the binary random alloy model) in the f.c.c. binary alloy as a function of composition $c_{A}$ with $w_{A} / w_{B}=$ $10^{-1}, 10^{-2}, 10^{-3}, 10^{-4}$. (For the Manning approach the maximum value of $S$ here is $1 / f_{0}$ i.e. 1.27967.)

where $s_{A}$ and $s_{B}$ are often called the partial vacancy-wind factors. These factors can be expressed in terms of the collective and tracer correlation factors as:

$$
s_{A}=\frac{f_{A A}-c_{A} f_{A B}^{(A)} c_{B}^{-1}}{f_{A}} ; \quad s_{B}=\frac{f_{B B}-c_{B} f_{A B}^{(B)} c_{A}^{-1}}{f_{B}}
$$

According to the Manning diffusion kinetics theory [1,2], the partial vacancy-wind factors can be expressed as:

$$
s_{A}=1+\frac{1-f_{0}}{f_{0}} \frac{c_{A}\left(D_{A}^{*}-D_{B}^{*}\right)}{c_{A} D_{A}^{*}+c_{B} D_{B}^{*}} ; \quad s_{B}=1+\frac{1-f_{0}}{f_{0}} \frac{c_{B}\left(D_{B}^{*}-D_{A}^{*}\right)}{c_{A} D_{A}^{*}+c_{B} D_{B}^{*}}
$$


In terms of the tracer correlation factors, the Manning diffusion kinetics theory gives the very simple expressions:

$$
s_{A}=\frac{f_{B}}{f_{0}}, \quad s_{B}=\frac{f_{A}}{f_{0}}
$$

In the Manning treatment, since the tracer correlation factors have the limits of zero and unity it is clear that the lower limit of the partial vacancy-wind factors is zero and that the upper limit is $f_{0}^{-1}$. For the corresponding treatment by the MAA self-consistent formalism there is no upper limit for the partial vacancy-wind factors (at the percolation threshold only) [9].

\section{Vacancy-Wind Factors in Interdiffusion in Ternary Alloys}

Some forty years were to pass before an analogous expression to the Darken-Manning Equation (Eq. 1) would be derived for relating interdiffusion and tracer diffusion coefficients in ternary and higher order alloys. Here, we confine ourselves to discussing the recent findings for ternary alloys. In the ternary alloy system $(A-B-C)$ for the matrix of the interdiffusion coefficients, the following analogue of the Darken-Manning Equation has been developed [10]:

$$
\left[\begin{array}{ll}
\widetilde{D}_{A A}^{C} & \widetilde{D}_{A B}^{C} \\
\widetilde{D}_{B A}^{C} & \widetilde{D}_{B B}^{C}
\end{array}\right]=\left[\begin{array}{ll}
S_{A A} \widetilde{d}_{A A} & S_{A B} \widetilde{d}_{A B} \\
S_{B A} \widetilde{d}_{B A} & S_{B B} \widetilde{d}_{B B}
\end{array}\right]\left[\begin{array}{cc}
\phi_{11} & \phi_{21} \\
\phi_{12} & \phi_{22}
\end{array}\right]
$$

where

$$
\begin{array}{ll}
\tilde{d}_{A A}=D_{A}^{*}-c_{A} D_{A}^{*}+c_{A} D_{C}^{*}, & \tilde{d}_{B A}=-c_{B} D_{A}^{*}+c_{B} D_{C}^{*} \\
\tilde{d}_{B B}=D_{B}^{*}-c_{B} D_{B}^{*}+c_{B} D_{C}^{*}, & \widetilde{d}_{A B}=-c_{A} D_{B}^{*}+c_{A} D_{C}^{*}
\end{array}
$$

and the vacancy-wind effect is now manifested as a matrix of vacancy-wind factors, the $S_{\mathrm{ij}}$. The very close structural similarity between Eq. 9, 10 for the ternary alloy and the well-known DarkenManning equation (Eq. 1) for the binary alloy should be especially noted. Approximations for the $S_{\mathrm{ij}}$ have also been developed very recently using a Manning-like treatment for the ternary random alloy [10]. In terms of the tracer diffusivities and composition the following closed-form expressions for the $S_{\mathrm{ij}}$ have been found:

$$
\begin{aligned}
& S_{A A}=1+\frac{2 c_{A}\left(c_{B}\left(D_{A}^{*}-D_{B}^{*}\right)+c_{C}\left(D_{A}^{*}-D_{C}^{*}\right)\right)\left(D_{A}^{*}-D_{C}^{*}\right)}{M_{0}\left(\left(1-c_{C}\right) D_{A}^{*}+c_{A} D_{C}^{*}\right)\left(c_{A} D_{A}^{*}+c_{B} D_{B}^{*}+c_{C} D_{C}^{*}\right)} \\
& S_{B B}=1+\frac{2 c_{B}\left(c_{A}\left(D_{B}^{*}-D_{A}^{*}\right)+c_{C}\left(D_{B}^{*}-D_{C}^{*}\right)\right)\left(D_{B}^{*}-D_{C}^{*}\right)}{M_{0}\left(\left(1-c_{C}\right) D_{B}^{*}+c_{B} D_{C}^{*}\right)\left(c_{A} D_{A}^{*}+c_{B} D_{B}^{*}+c_{C} D_{C}^{*}\right)} \\
& S_{A B}=1+\frac{2}{M_{0}}\left(1-\frac{D_{A}^{*}}{c_{A} D_{A}^{*}+c_{B} D_{B}^{*}+c_{C} D_{C}^{*}}\right) \\
& S_{B A}=1+\frac{2}{M_{0}}\left(1-\frac{D_{B}^{*}}{c_{A} D_{A}^{*}+c_{B} D_{B}^{*}+c_{C} D_{C}^{*}}\right)
\end{aligned}
$$


The diagonal terms $S_{\mathrm{AA}}$ and $S_{\mathrm{BB}}$ can be reduced to the vacancy-wind factor $S$ (given by the generic Eq. 3) for the corresponding binary alloy system with $c_{\mathrm{B}}=0$ (for an $\mathrm{A}-\mathrm{C}$ binary alloy) or $c_{\mathrm{A}}=0$ (for a B-C binary alloy) respectively.

In terms of the tracer correlation factors $f_{\mathrm{A}}, f_{\mathrm{B}}$ and $f_{\mathrm{C}}$ for the three atomic components the following expressions for the vacancy-wind factors can be derived (these expressions are formally analogous to Eq. 4):

$$
\begin{aligned}
S_{B A} & =\frac{f_{B}}{f_{0}} \quad S_{A B}=\frac{f_{A}}{f_{0}} \\
S_{A A} & =\frac{\left(f_{A} / f_{0}\right) f_{C} c_{A}\left(w_{A}-w_{C}\right)-w_{A}}{f_{C} c_{A}\left(w_{A}-w_{C}\right)-w_{A}} \\
S_{B B} & =\frac{\left(f_{B} / f_{0}\right) f_{C} c_{B}\left(w_{B}-w_{C}\right)-w_{B}}{f_{C} c_{B}\left(w_{B}-w_{C}\right)-w_{B}}
\end{aligned}
$$

The lower limit of the off-diagonal vacancy-wind factors is zero and the upper limit is $1 / f_{0}$. By direct inspection, the diagonal terms (for the compositions $\mathrm{c}_{A}, \mathrm{c}_{B}$ and $\mathrm{c}_{C}>0.1$, e.g. for non-dilute alloys) we have that the upper limit is slightly higher than $1 / f_{0}$ and the lower limit is very close to unity.

The MAA approximation has also been applied to the determination of the $S_{\mathrm{ij}}$ in the ternary random alloy system. As for the binary random alloy, this again requires a numerical approach [10]. It was found that the behaviour of the diagonal vacancy-wind elements of the ternary alloy is similar to the behaviour of the vacancy-wind factor for the binary system. Namely, the matrix elements corresponding to the most mobile species can be relatively high at the points where the composition of this component is about $1-f_{0}$. The lower bound for the diagonal factors is about 0.8 . There are rather substantial differences between the results from Manning and MAA but in general the two approaches give quite similar results when the vacancy atom exchange frequencies are relatively close, within a factor of five.

It is appropriate here to present some ternary alloy vacancy-wind factors calculated from the Manning approach and MAA that have been determined from experimental data. We consider the ternary alloy system $\mathrm{Cu}-\mathrm{Fe}-\mathrm{Ni}$. All three tracer diffusivities are available at five composition points at the temperature $\mathrm{T}=1271 \mathrm{~K}$ [11]. The resulting vacancy-wind factors are presented in Table 1 using the Manning approach (Eqs. 10 and 11) and in Table 2 using the MAA approach. 


\begin{tabular}{|c|c|c|c|c|c|c|}
\hline $\mathrm{c}_{\mathrm{Fe}}$ & $\mathrm{c}_{\mathrm{Ni}}$ & $\mathrm{c}_{\mathrm{Cu}}$ & $S_{\mathrm{CuCu}}$ & $S_{\mathrm{FeFe}}$ & $S_{\mathrm{CuFe}}$ & $S_{\mathrm{FeCu}}$ \\
\hline 0.127 & 0.686 & 0.187 & 1.09 & 1.01 & 0.52 & 0.91 \\
\hline 0.265 & 0.503 & 0.232 & 1.12 & 0.99 & 0.52 & 1.06 \\
\hline 0.108 & 0.464 & 0.428 & 1.19 & 0.99 & 0.71 & 1.12 \\
\hline 0.298 & 0.375 & 0.327 & 1.06 & 1.02 & 0.83 & 0.93 \\
\hline 0.105 & 0.802 & 0.093 & 1.01 & 1.04 & 0.82 & 0.53 \\
\hline
\end{tabular}

Table 1 . The vacancy-wind factor matrix elements $\left(S_{i j}\right)$ calculated using the Manning-type approach (Eqs. 10 and 11) from the measured tracer diffusivities [11] in the $\mathrm{Cu}-\mathrm{Fe}-\mathrm{Ni}$ system.

\begin{tabular}{|l|l|l|l|l|l|l|}
\hline $\mathrm{c}_{\mathrm{Fe}}$ & $\mathrm{c}_{\mathrm{Ni}}$ & $\mathrm{c}_{\mathrm{Cu}}$ & $S_{\mathrm{CuCu}}$ & $S_{\mathrm{FeFe}}$ & $S_{\mathrm{CuFe}}$ & $S_{\mathrm{FeCu}}$ \\
\hline 0.127 & 0.686 & 0.187 & 1.22 & 0.99 & -0.11 & 0.84 \\
\hline 0.265 & 0.503 & 0.232 & 1.37 & 0.96 & 0.02 & 1.27 \\
\hline 0.108 & 0.464 & 0.428 & 1.45 & 0.97 & 0.46 & 1.34 \\
\hline 0.298 & 0.375 & 0.327 & 1.11 & 1.03 & 0.72 & 0.89 \\
\hline 0.105 & 0.802 & 0.093 & 1.01 & 1.10 & 0.63 & -0.04 \\
\hline
\end{tabular}

Table 2. The vacancy-wind factor matrix elements $\left(S_{i j}\right)$ calculated using the MAA-type approach from the measured tracer diffusivities [11] in the $\mathrm{Cu}-\mathrm{Fe}-\mathrm{Ni}$ system.

For the diagonal elements in the $S_{\mathrm{ij}}$ matrix in general, we can see that the use of the Manning formalism can produce quite a significant discrepancy (compared with the use of the MAA approach which is known to provide an almost exact solution). In the $\mathrm{Cu}-\mathrm{Fe}-\mathrm{Ni}$ system, for the available data, the maximum discrepancy is about $20 \%$. However, the discrepancy can be much larger in other systems [10]. The situation with the off-diagonal vacancy-wind factors is quite complicated. It is clear that the Manning approach gives estimates that are reliable only if they are close to unity. If the Manning approach gives estimates that are significantly lower than unity, then the corresponding improvement by MAA can be quite significant and often puts the vacancy-wind factor well below unity (or even change its sign). If the Manning approach gives values that are higher than unity then the improved MAA estimate might be different, but it will still be higher than unity. These findings concerning the off-diagonal vacancy-wind factors are valid in general for ternary alloys. 


\section{Vacancy-Wind Factors in Ionic Compounds (Two Conducting Ions)}

A vacancy-wind effect occurs in the d.c. ionic conductivity in systems where two or more ions (usually cations) share the same sublattice and compete for the same vacancies. Considering the partial d.c. ionic conductivities and assuming for convenience the same charge state $q$ on each cation $(\mathrm{A}, \mathrm{B})$ we write that:

$$
\sigma_{A}=\frac{C_{A} q^{2} D_{A}^{*} p_{A}}{k T} \quad \text { and } \quad \sigma_{B}=\frac{C_{B} q^{2} D_{B}^{*} p_{B}}{k T}
$$

where the $p_{\mathrm{A}(\mathrm{B})}$ are called partial vacancy-wind factors, $C_{\mathrm{A}(\mathrm{B})}$ is the concentration of $\mathrm{A}(\mathrm{B})$ and $k$ and $T$ are the Boltzmann constant and absolute temperature respectively. These particular partial vacancy-wind factors have also been called "physical correlation factors" in the past, especially in the fast ion transport literature [12]. The partial ionic conductivity vacancy-wind factors can be expressed exactly in terms of the tracer and collective correlation factors:

$$
p_{A}=\frac{f_{A A}+f_{A B}^{(A)}}{f_{A}} \text { and } p_{B}=\frac{f_{B B}+f_{A B}^{(B)}}{f_{B}}
$$

When the Manning diffusion kinetics formalism for the random alloy is applied, we obtain the very simple result:

$$
p_{A}=p_{B}=\frac{1}{f_{0}}
$$

This means that the vacancy-wind effect in this situation of randomly mixed ions enhances the partial ionic conductivities of both cations equally and with a constant factor.

The principal method for determining partial ionic conductivities is the quite difficult to perform 'Chemla experiment' [13]. The total ionic conductivity $\sigma$ of the mixed system is, however, straightforward to measure and should be the natural quantity to focus on here. The expression relating the total ionic conductivity to the individual tracer diffusion coefficients is:

$$
\sigma=\sigma_{A}+\sigma_{B}=\frac{q^{2}}{k T} P\left(C_{A} D_{A}^{*}+C_{B} D_{B}^{*}\right)
$$

where $P$ is the ionic conductivity vacancy-wind factor and $q$ is the charge on the cations (assumed here to be the same). It is also worth noting that $P$ as presented in Eq. 18 has been used as a definition of (the reciprocal) of the Haven Ratio in mixed cation conductors such as glasses [14]. $P$ can be expressed in terms of the collective and tracer correlation factors:

$$
P=\frac{C_{A} f_{A A}+2 C_{A} f_{A B}^{(A)}+C_{B} g f_{B B}}{C_{A} f_{A}+C_{B} g f_{B}}
$$


Manning calculated the vacancy-wind factor $P$ only for the five-frequency model [1]. $P$ has been calculated for interacting systems for the purposes of modelling mixed solid state electrolytes [12]. The random mixing (random 'alloy') model is also useful to employ here. Using the Manning diffusion kinetics formalism for the random alloy we find that the vacancy-wind factor $P$ is simply equal to $p_{\mathrm{A}}$ and $p_{\mathrm{B}}$ and therefore:

$$
P=\frac{1}{f_{0}}
$$

Note that this result (and Eq. 17) is only valid for the Manning diffusion kinetics formalism for the random alloy and in the very dilute vacancy limit where this theory is framed. When the MAA diffusion kinetics formalism for the random alloy is used to calculate $P$, the behaviour as shown in Fig. 2 is found, which is clearly quite different from the Manning result. However, when the vacancy atom exchange frequencies are within about a factor of five, there is little difference between MAA and Manning.

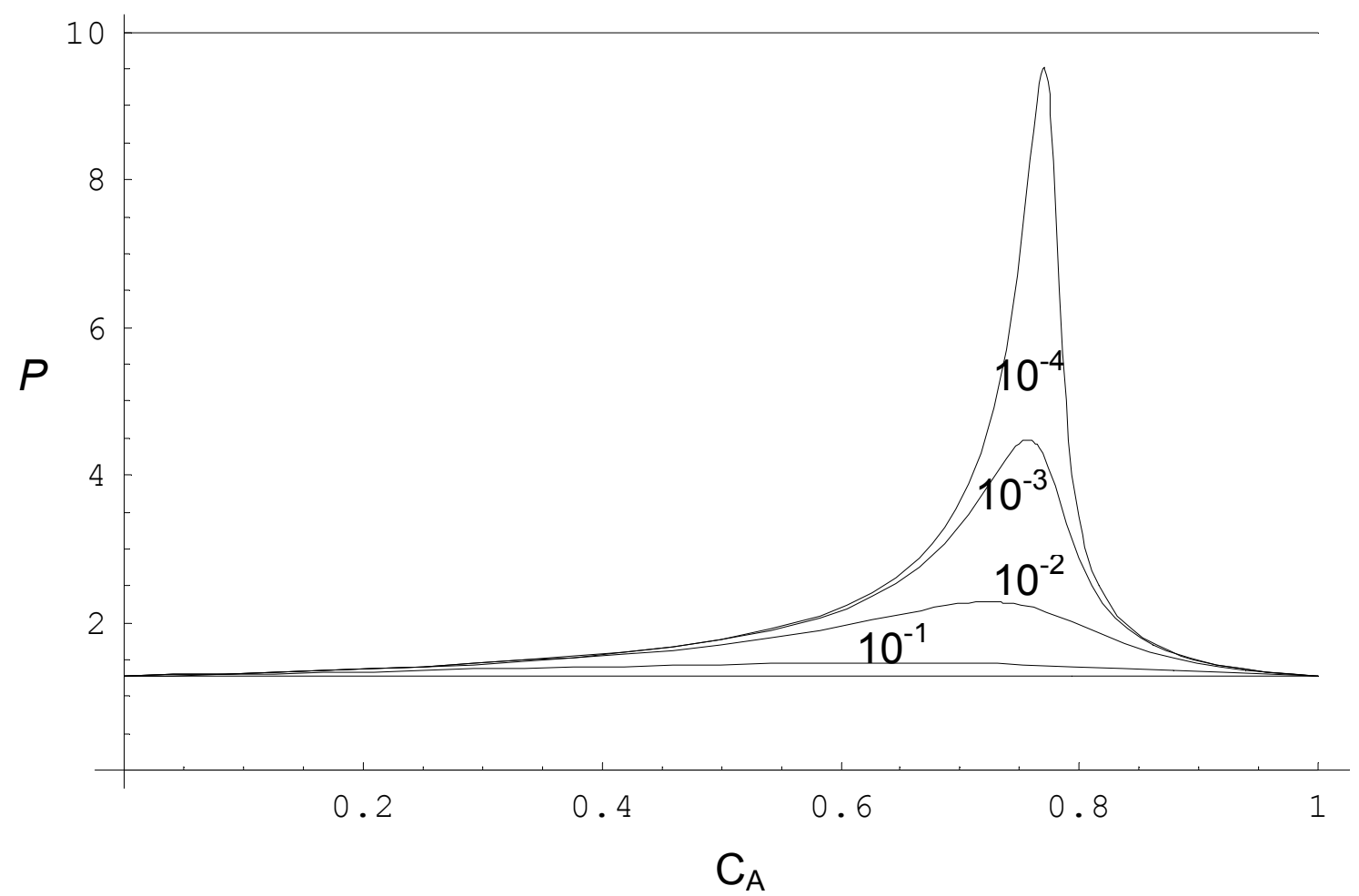

Figure 2. The vacancy-wind factor $P$ (calculated using the MAA diffusion kinetics theory for the binary random alloy model) in the f.c.c. binary alloy as a function of composition $c_{A}$ with $w_{A} / w_{B}=$ $10^{-1}, 10^{-2}, 10^{-3}, 10^{-4}$. (For the Manning approach $P$ is $1 / f_{0}$ i.e. 1.27967 .) 


\section{Apparent Vacancy-Wind Factors in Interdiffusion in Insulating Ionic Crystals}

In cation interdiffusion in mixed-cation ionic crystals (with the cations having the same charge state) there are several limiting cases to consider. If there is abundant electronic conductivity then it is still appropriate to use the Darken-Manning Equation (Eq. 1). On the other hand, if the crystals are highly insulating and the mobility of the anions is very low compared with the cations (examples would be interdiffusion in some mixed cation silicates and carbonates with fixed valence cations), then the correct equation to use in order to relate the interdiffusion coefficient to the tracer diffusion coefficients is what is popularly known in the solid state literature as the Nernst-Planck Equation, though strictly it is the Nernst-Hartley Equation:

$$
\widetilde{D}=\frac{D_{A^{*}} D_{B^{*}}}{c_{A} D_{A^{*}}+c_{B} D_{B^{*}}} \phi
$$

Interestingly, the Manning diffusion kinetics formalism for a randomly mixed system (the random alloy) is fully consistent with Eq. 21 as it stands. The Manning formalism gives no extra factor in Eq. 21 that might be analogous to a vacancy-wind factor. However, when the near-exact MAA formalism for the random alloy is applied to the problem we do in fact find a non-trivial extra factor that we have termed $S^{\mathrm{NP}}$ that needs to be added to Eq. 21 [15], i.e.

$$
\widetilde{D}=\frac{D_{A^{*}} D_{B^{*}}}{c_{A} D_{A^{*}}+c_{B} D_{B^{*}}} S^{N P} \phi
$$

where the extra factor $S^{\mathrm{NP}}$ is described formally in terms of tracer and collective correlation factors by:

$$
S^{N P}=\frac{M_{0}}{2}\left(\frac{f_{A B}^{(A)}}{f_{A}}+\frac{f_{A B}^{(B)}}{f_{B}}\right)
$$

The Manning diffusion kinetics formalism in effect puts $S^{\mathrm{NP}}$ equal to unity. Fig. 3 shows the behaviour determined by MAA of $S^{\mathrm{NP}}$ for a range of ratios of the vacancy-atom exchange frequencies in the f.c.c. random alloy. In its formal presentation in Eq. 22, $S^{\mathrm{NP}}$ has the general appearance of a vacancy-wind factor. Moreover, it is related to off-diagonal phenomenological coefficients as can be quite clearly seen in Eq. 23. However, in interdiffusion of cations (of the same charge) of insulating ionic crystals with immobile anions there is no net vacancy flux. Nonetheless, there could in principle be local vacancy fluxes but the details how these might actually operate remain unknown. The physical processes leading to $S^{\mathrm{NP}}$ are presently being investigated by the authors using direct simulation methods. 


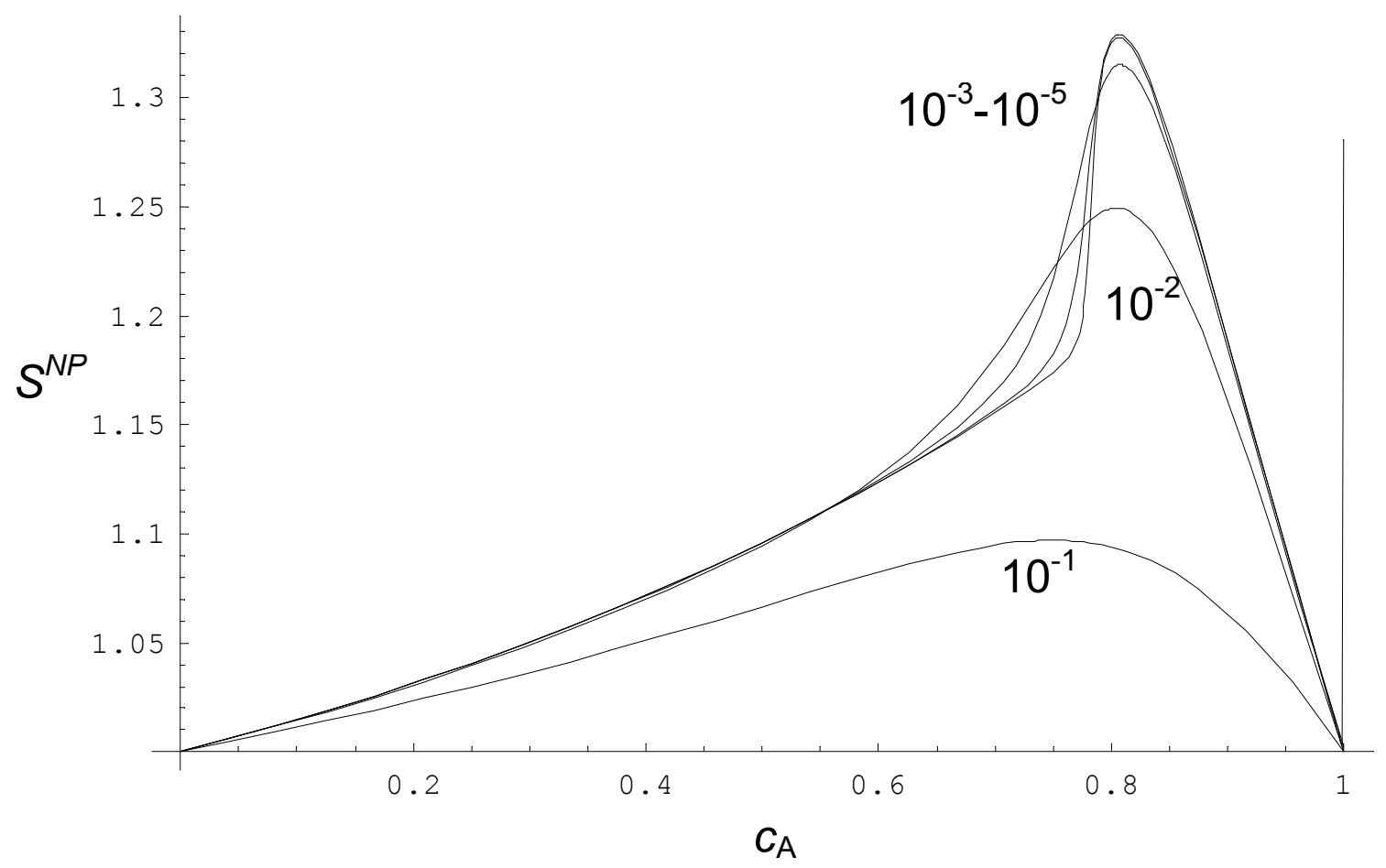

Figure 3. The factor $S^{N P}$ (calculated using the MAA diffusion kinetics theory for the binary random mixing model) in the f.c.c. lattice as a function of composition $c_{A}$ with $w_{A} / w_{B}=10^{-1}-10^{-5}$. (For the Manning diffusion kinetics theory $S^{N P}=1$.)

\section{Conclusions}

In this paper, we have reviewed recent progress in developing accurate new expressions for the vacancy-wind factors occurring in various relations between collective transport quantities and tracer diffusion coefficients of the mobile components. We have mainly focused on concentrated systems, where the random alloy model is appropriate. Processes covered were chemical diffusion/tracer diffusion in binary and ternary alloys, ionic conductivity/tracer diffusion and chemical diffusion/tracer diffusion in mixed fixed valence cation ionic conductors.

\section{Acknowledgements}

We wish to thank the Australian Research Council for its support of this research under the Discovery Project Grants Scheme.

\section{References}

[1] J.R. Manning, Diffusion Kinetics for Atoms in Crystals (Princeton, New Jersey: Van Nostrand) 1968.

[2] J.R. Manning, Phys. Rev. B, Vol. 4 (1971), p. 1111

[3] G.E. Murch and R.J. Thorn, Phil. Mag. A, Vol. 39 (1979), p. 259.

[4] L.S. Darken, Trans. AIME, Vol. 180 (1948), p.430.

[5] G.E. Murch and I.V. Belova, Phil. Mag. A, Vol. 81 (2001), p. 83.

[6] A.R. Allnatt and E.L. Allnatt, Phil. Mag. A, Vol. 49, (1984), p. 625.

[7] I.V. Belova and G.E. Murch, Defect Diffus. Forum, Vols. 237-240 (2005), p. 291 (2005).

[8] L.K. Moleko, A.R. Allnatt and E.L. Allnatt, Phil. Mag. A, Vol. 59 (1989), p. 141. 
[9] I.V. Belova and G.E. Murch, Phil. Mag. A, Vol. 81 (2001), p. 1749

[10] I.V. Belova and G.E. Murch, Acta Mat., Vol. 55 (2007), p. 627.

[11] S.V. Divinski, F. Hisker, Chr. Herzig, R. Filipek, M. Danieleweski, Defect Diffus. Forum, Vols 237-240 (2005), p. 50.

[12] A. Suzuki, H. Sato and R. Kikuchi, Phys. Rev. B, Vol. 29 (1984), p. 3550.

[13] M. Chemla, Ann. Phys., Paris, Vol. 13 (1956), p. 959.

]14] A.W. Imre, S. Voss and H. Mehrer, J. Non-Cryst. Solids, Vol. 333 (2004), p. 231.

[15] I.V. Belova A.R. Allnatt and G.E. Murch, Phil. Mag. accepted for publication and in press. 
Diffusion in Advanced Materials and Processing

doi:10.4028/3-908451-45-0

Non-Random Interaction of Vacancies with Atoms during Interdiffusion and lonic Conductivity in Materials

doi:10.4028/3-908451-45-0.119 